\title{
Expansion formulas for an extended Hurwitz-Lerch zeta function obtained via fractional calculus
}

Hari M Srivastava', Sébastien Gaboury ${ }^{2 *}$ and Abdelmejid Bayad ${ }^{3}$

"Correspondence:

s1gabour@uqac.ca

${ }^{2}$ Department of Mathematics and

Computer Science, University of

Québec at Chicoutimi, Chicoutimi,

Québec G7H 2B1, Canada

Full list of author information is

available at the end of the article

\begin{abstract}
Motivated by the recent investigations of several authors, in this paper, we derive several new expansion formulas involving a generalized Hurwitz-Lerch zeta function introduced and studied recently by Srivastava et al. (Integral Transforms Spec. Funct. 22:487-506, 2011). These expansions are obtained by using some fractional calculus theorems such as the generalized Leibniz rules for the fractional derivatives and the Taylor-like expansions in terms of different functions. Several (known or new) special cases are also considered.
\end{abstract}

MSC: Primary 11M25; 11M35; 26A33; secondary 33C05; 33C60

Keywords: fractional derivatives; generalized Taylor expansion; generalized Hurwitz-Lerch zeta functions; Riemann zeta function; Leibniz rules

\section{Introduction}

The Hurwitz-Lerch zeta function $\Phi(z, s, a)$ is defined by (see, for example, [1, p.121 et seq.]; see also [2] and [3, p.194 et seq.])

$$
\begin{aligned}
& \Phi(z, s, a):=\sum_{n=0}^{\infty} \frac{z^{n}}{(n+a)^{s}} \\
&\left(a \in \mathbb{C} \backslash \mathbb{Z}_{0}^{-} ; s \in \mathbb{C} \text { when }|z|<1 ; \Re(s)>1 \text { when }|z|=1\right) .
\end{aligned}
$$

The Hurwitz-Lerch zeta function contains, as its special cases, the Riemann zeta function $\zeta(s)$, the Hurwitz zeta function $\zeta(s, a)$, and the Lerch zeta function $\ell_{s}(\xi)$ defined by

$$
\begin{aligned}
& \zeta(s):=\sum_{n=1}^{\infty} \frac{1}{n^{s}}=\Phi(1, s, 1)=\zeta(s, 1) \quad(\Re(s)>1), \\
& \zeta(s, a):=\sum_{n=0}^{\infty} \frac{1}{(n+a)^{s}}=\Phi(1, s, a) \quad\left(\Re(s)>1 ; a \in \mathbb{C} \backslash \mathbb{Z}_{0}^{-}\right)
\end{aligned}
$$

and

$$
\ell_{s}(\xi):=\sum_{n=1}^{\infty} \frac{\mathrm{e}^{2 n \pi \mathrm{i} \xi}}{(n+1)^{s}}=\Phi\left(\mathrm{e}^{2 \pi \mathrm{i} \xi}, s, 1\right) \quad(\Re(s)>1 ; \xi \in \mathbb{R}),
$$

respectively.

\section{Springer}

(c) 2014 Srivastava et al.: licensee Springer. This is an Open Access article distributed under the terms of the Creative Commons Attribution License (http://creativecommons.org/licenses/by/2.0), which permits unrestricted use, distribution, and reproduction in any medium, provided the original work is properly cited. 
The Hurwitz-Lerch zeta function $\Phi(z, s, a)$ defined in (1.5) can be continued meromorphically to the whole complex $s$-plane, except for a simple pole at $s=1$ with its residue 1 . It is well known that

$$
\begin{aligned}
\Phi(z, s, a) & =\frac{1}{\Gamma(s)} \int_{0}^{\infty} \frac{t^{s-1} \mathrm{e}^{-a t}}{1-z \mathrm{e}^{-t}} \mathrm{~d} t \\
(\Re(a) & >0 ; \Re(s)>0 \text { when }|z| \leqq 1(z \neq 1) ; \Re(s)>1 \text { when } z=1) .
\end{aligned}
$$

It is worth noting that the Hurwitz-Lerch zeta function $\Phi(z, s, a)$ defined in (1.5) is also related to several families of special polynomials such as the Bernoulli, the Euler, and the Genocchi polynomials [3-5].

Recently, a more general family of Hurwitz-Lerch zeta functions was investigated by Lin and Srivastava [6, p.727, Eq. (8)]. Srivastava and Lin studied the following function:

$$
\begin{aligned}
& \Phi_{\mu, \nu}^{(\rho, \sigma)}(z, s, a):=\sum_{n=0}^{\infty} \frac{(\mu)_{\rho n}}{(\nu)_{\sigma n}} \frac{z^{n}}{(a+n)^{s}} \\
& \left(\mu \in \mathbb{C} ; a, \nu \in \mathbb{C} \backslash \mathbb{Z}_{0}^{-} ; \rho, \sigma \in \mathbb{R}^{+} ; \rho<\sigma \text { when } s, z \in \mathbb{C} ;\right. \\
& \rho=\sigma \text { and } s \in \mathbb{C} \text { when }|z|<1 ; \rho=\sigma \text { and } \Re(s-\mu+\nu)>1 \text { when }|z|=1) .
\end{aligned}
$$

Here, and for the remainder of this paper, $(\lambda)_{\kappa}$ denotes the Pochhammer symbol defined, in terms of the gamma function, by

$$
(\lambda)_{\kappa}:=\frac{\Gamma(\lambda+\kappa)}{\Gamma(\lambda)}= \begin{cases}1 & (\kappa=0 ; \lambda \in \mathbb{C} \backslash\{0\}), \\ \lambda(\lambda+1) \cdots(\lambda+n-1) & (\kappa=n \in \mathbb{N} ; \lambda \in \mathbb{C}),\end{cases}
$$

it being understood conventionally that $(0)_{0}:=1$ and assumed tacitly that the $\Gamma$-quotient exists (see, for details, [7, p.21 et seq.]).

Clearly, we find from the definition (1.6) that

$$
\Phi_{v, v}^{(\sigma, \sigma)}(z, s, a)=\Phi_{\mu, \nu}^{(0,0)}(z, s, a)=\Phi(z, s, a)
$$

and

$$
\begin{aligned}
& \Phi_{\mu, 1}^{(1,1)}(z, s, a)=\Phi_{\mu}^{*}(z, s, a):=\sum_{n=0}^{\infty} \frac{(\mu)_{n}}{n !} \frac{z^{n}}{(n+a)^{s}} \\
& \left(\mu \in \mathbb{C} ; a, v \in \mathbb{C} \backslash \mathbb{Z}_{0}^{-} ; s \in \mathbb{C} \text { when }|z|<1 ; \Re(s-\mu)>1 \text { when }|z|=1\right),
\end{aligned}
$$

where the function $\Phi_{\mu}^{*}(z, s, a)$ involved in (1.9) is a generalization of the Hurwith-Lerch zeta function considered by Goyal and Laddha [8, p.100, Eq. (1.5)].

A generalization of the above-defined Hurwitz-Lerch zeta functions $\Phi(z, s, a)$ and $\Phi_{\mu}^{*}(z, s, a)$ was studied by Garg et al. [9, p.313, Eq. (1.7)] in the following form:

$$
\begin{aligned}
& \Phi_{\lambda, \mu ; \nu}(z, s, a):=\sum_{n=0}^{\infty} \frac{(\lambda)_{n}(\mu)_{n}}{(\nu)_{n} n !} \frac{z^{n}}{(n+a)^{s}} \\
&\left(\lambda, \mu \in \mathbb{C} ; \nu, a \in \mathbb{C} \backslash \mathbb{Z}_{0}^{-} ; s \in \mathbb{C} \text { when }|z|<1 ; \Re(s+\nu-\lambda-\mu)>1 \text { when }|z|=1\right) .
\end{aligned}
$$


Srivastava et al. [10, p.491, Eq. (1.20)] (see also [11-13]), in the year 2011, considered a further generalization of the Hurwitz-Lerch zeta function, defined in the form

$$
\begin{aligned}
& \Phi_{\lambda, \mu ; \nu}^{(\rho, \sigma, \kappa)}(z, s, a):=\sum_{n=0}^{\infty} \frac{(\lambda)_{\rho n}(\mu)_{\sigma n}}{(\nu)_{\kappa n} n !} \frac{z^{n}}{(n+a)^{s}} \\
& \left(\lambda, \mu \in \mathbb{C} ; a, \nu \in \mathbb{C} \backslash \mathbb{Z}_{0}^{-} ; \rho, \sigma, \kappa \in \mathbb{R}^{+} ; \kappa-\rho-\sigma>-1 \text { when } s, z \in \mathbb{C} ;\right. \\
& \kappa-\rho-\sigma=-1 \text { and } s \in \mathbb{C} \text { when }|z|<\delta^{*}:=\rho^{-\rho} \sigma^{-\sigma} \kappa^{\kappa} ; \\
& \left.\kappa-\rho-\sigma=-1 \text { and } \Re(s+\nu-\lambda-\mu)>1 \text { when }|z|=\delta^{*}\right) .
\end{aligned}
$$

Several integral representations, relationships with the $\bar{H}$-function, fractional derivatives, and analytic continuation formulas were established for the function defined in (1.11).

It is worth noting the following special or limit cases of the function $\Phi_{\lambda, \mu ; v}^{(\rho, \sigma, \kappa)}(z, s, a)$.

(i) For $\lambda=\rho=1$, we find that

$$
\Phi_{1, \mu ; \nu}^{(1, \sigma, \kappa)}(z, s, a)=\Phi_{\mu ; \nu}^{(\sigma, \kappa)}(z, s, a)
$$

in terms of the generalized Hurwitz-Lerch zeta function $\Phi_{\mu ; v}^{(\sigma, \kappa)}(z, s, a)$ defined in (1.6).

(ii) If we set $\rho=\sigma=\kappa=1$, then (1.11) yields the generalized Hurwitz-Lerch zeta function $\Phi_{\lambda, \mu ; \nu}(z, s, a)$ studied by Garg et al. [9] and Jankov et al. [14]:

$$
\Phi_{\lambda, \mu ; \nu}^{(1,1,1)}(z, s, a)=\Phi_{\lambda, \mu ; \nu}(z, s, a)
$$

(iii) Setting $\rho=\sigma=\kappa=1$ and $\lambda=v$, (1.11) reduces to the function $\Phi_{\mu}^{*}(z, s, a)$ investigated by Goyal and Laddha [8] as below:

$$
\Phi_{\nu, \mu ; \nu}^{(1,1,1)}(z, s, a)=\Phi_{\mu}^{*}(z, s, a)
$$

(iv) In (1.11), we put $\mu=\rho=\sigma=1$ and $z \mapsto \frac{z}{\lambda}$. Then, by the familiar principle of confluence, the limit case when $\lambda \rightarrow \infty$, would yield the Mittag-Leffler type function $E_{\kappa, v}^{(a)}(s, z)$ studied by Barnes [15], namely

$$
\begin{aligned}
& \lim _{\lambda \rightarrow \infty}\left\{\frac{1}{\Gamma(\nu)} \Phi_{\lambda, 1 ; \nu}^{(1,1, \kappa)}\left(\frac{z}{\lambda}, s, a\right)\right\}=\sum_{n=0}^{\infty} \frac{z^{n}}{\Gamma(\nu+\kappa n)(n+a)^{s}}:=E_{\kappa, \nu}^{(a)}(s ; z) \\
& \left(a, v \in \mathbb{C} \backslash \mathbb{Z}_{0}^{-} ; \Re(\kappa)>0 ; s, z \in \mathbb{C}\right) .
\end{aligned}
$$

(v) A limit case of the generalized Hurwitz-Lerch function $\Phi_{\lambda, \mu ; v}^{(\rho, \sigma, \kappa)}(z, s, a)$, which is of interest in our present investigation, is given by

$$
\begin{aligned}
& \Phi_{\mu ; \nu}^{*(\sigma, \kappa)}(z, s, a):=\lim _{|\lambda| \rightarrow \infty}\left\{\Phi_{\lambda, \mu ; \nu}^{(\rho, \sigma, \kappa)}\left(\frac{z}{\lambda^{\rho}}, s, a\right)\right\}=\sum_{n=0}^{\infty} \frac{(\mu)_{\sigma n}}{(\nu)_{\kappa n} n !} \frac{z^{n}}{(n+a)^{s}} \\
& \left(\mu \in \mathbb{C} ; a, v \in \mathbb{C} \backslash \mathbb{Z}_{0}^{-} ; \sigma, \kappa \in \mathbb{R}^{+} ; s \in \mathbb{C} \text { when }|z|<\sigma^{-\sigma} \kappa^{\kappa} ;\right. \\
& \left.\Re(s+\nu-\mu)>1 \text { when }|z|=\sigma^{-\sigma} \kappa^{\kappa}\right) .
\end{aligned}
$$


(vi) Another limit case of the generalized Hurwitz-Lerch function $\Phi_{\lambda, \mu ; \nu}^{(\rho, \sigma, \kappa)}(z, s, a)$ is given by

$$
\begin{gathered}
\Phi_{\mu}^{*(\sigma)}(z, s, a):=\lim _{\min \{|\lambda|,|v|\} \rightarrow \infty}\left\{\Phi_{\lambda, \mu ; \nu}^{(\rho, \sigma, \kappa)}\left(\frac{z \nu^{\kappa}}{\lambda^{\rho}}, s, a\right)\right\}=\sum_{n=0}^{\infty} \frac{(\mu)_{\sigma n}}{n !} \frac{z^{n}}{(n+a)^{s}} \\
\left(\mu \in \mathbb{C} ; a \in \mathbb{C} \backslash \mathbb{Z}_{0}^{-} ; 0<\sigma<1 \text { and } s, z \in \mathbb{C} ; \sigma=1 \text { and } s \in \mathbb{C}\right. \\
\text { when } \left.|z|<\sigma^{-\sigma} ; \sigma=1 \text { and } \Re(s-\mu)>1 \text { when }|z|=\sigma^{-\sigma}\right),
\end{gathered}
$$

which, for $\sigma=1$, reduces at once to the function $\Phi_{\mu}^{*}(z, s, a)$ defined by (1.9).

Finally, a multiparameter extension of the function $\Phi_{\lambda, \mu ; \nu}^{(\rho, \kappa)}(z, s, a)$ was given, more recently, by Srivastava et al. [10] (see also [12]). They considered the following function:

$$
\begin{aligned}
& \Phi_{\lambda_{1}, \ldots, \lambda_{p} ; \mu_{1}, \ldots, \mu_{q}}^{\left(\rho_{1}, \ldots, \rho_{p}, \sigma_{1}, \ldots, \sigma_{q}\right)}(z, s, a):=\sum_{n=0}^{\infty} \frac{\prod_{j=1}^{p}\left(\lambda_{j}\right)_{n \rho_{j}}}{n ! \prod_{j=1}^{q}\left(\mu_{j}\right)_{n \sigma_{j}}} \frac{z^{n}}{(n+a)^{s}} \\
& \left(p, q \in \mathbb{N}_{0} ; \lambda_{j} \in \mathbb{C}(j=1, \ldots, p) ; a, \mu_{j} \in \mathbb{C} \backslash \mathbb{Z}_{0}^{-}(j=1, \ldots, q) ;\right. \\
& \rho_{j}, \sigma_{k} \in \mathbb{R}^{+}(j=1, \ldots, p ; k=1, \ldots, q) ; \\
& \Delta>-1 \text { when } s, z \in \mathbb{C} ; \Delta=-1 \text { and } s \in \mathbb{C} \text { when } \\
& \left.|z|<\nabla^{*} ; \Delta=-1 \text { and } \Re(\Xi)>\frac{1}{2} \text { when }|z|=\nabla^{*}\right)
\end{aligned}
$$

with

$$
\begin{aligned}
& \nabla^{*}:=\left(\prod_{j=1}^{p} \rho_{j}^{-\rho_{j}}\right) \cdot\left(\prod_{j=1}^{q} \sigma_{j}^{\sigma_{j}}\right), \\
& \Delta:=\sum_{j=1}^{q} \sigma_{j}-\sum_{j=1}^{p} \rho_{j} \text { and } \Xi:=s+\sum_{j=1}^{q} \mu_{j}-\sum_{j=1}^{p} \lambda_{j}+\frac{p-q}{2} .
\end{aligned}
$$

It is fairly straightforward to see that if we let $p-1=q=1$ in (1.18), then we obtain the generalized Hurwitz-Lerch zeta function $\Phi_{\lambda, \mu ; \nu}^{(\rho, \sigma)}(z, s, a)$.

The aim of this paper is to extend several interesting results obtained recently by Gaboury and Bayad [16] and by Gaboury [17] to the Hurwitz-Lerch zeta function $\Phi_{\lambda, \mu ; \nu}^{(\rho, \kappa)}(z$, $s, a$ ) introduced and studied by Srivastava et al. [10]. This paper is organized as follows. Section 2 is devoted to the representation of the fractional derivatives based on Pochhammer's contour of integration. In Section 3, we recall some major fractional calculus theorems, that is, two generalized Leibniz rules and three Taylor-like expansions. Section 4 is dedicated to the proofs of the main results and, finally, Section 5 aims to provide some (new or known) special cases.

\section{Pochhammer contour integral representation for fractional derivative}

The fractional derivative of arbitrary order $\alpha, \alpha \in \mathbb{C}$, is an extension of the familiar $n$th derivative $D_{g(z)}^{n} F(z)=d^{n} F(z) /(d g(z))^{n}$ of the function $F(z)$ with respect to $g(z)$ to nonintegral values of $n$ and denoted by $D_{g(z)}^{\alpha} F(z)$. The aim of this concept is to generalize 
classical results of the $n$th order derivative to fractional order. Most of the properties of the classical calculus have been expanded to fractional calculus. For instance, the composition rule, the Leibniz rule, the chain rule and the Taylor and Laurent series. Fractional calculus provides tools that make easier to deal with special functions of mathematical physics. Many examples of the use of fractional derivatives appear in the literature: ordinary and partial differential equations, integral equations, integro-differential equations of non-integer order. Many other applications have been investigated through various field of science and engineering. For more details on fractional calculus, the reader could read [18-21].

The most familiar representation for the fractional derivative of order $\alpha$ of $z^{p} f(z)$ is the Riemann-Liouville integral [19] (see also [22-24]), that is,

$$
\mathcal{D}_{z}^{\alpha}\left\{z^{p} f(z)\right\}=\frac{1}{\Gamma(-\alpha)} \int_{0}^{z} f(\xi) \xi^{p}(\xi-z)^{-\alpha-1} \mathrm{~d} \xi \quad(\Re(\alpha)<0 ; \Re(p)>1),
$$

where the integration is carried out along a straight line from 0 to $z$ in the complex $\xi$-plane. By integrating by part $m$ times, we obtain

$$
\mathcal{D}_{z}^{\alpha}\left\{z^{p} f(z)\right\}=\frac{\mathrm{d}^{m}}{\mathrm{~d} z^{m}}\left\{\mathcal{D}_{z}^{\alpha-m}\left\{z^{p} f(z)\right\}\right\} .
$$

This allows us to modify the restriction $\Re(\alpha)<0$ to $\Re(\alpha)<m$ (see [24]).

Another representation for the fractional derivative is based on the Cauchy integral formula. This representation, too, has been widely used in many interesting papers (see, for example, the work of Osler [25-28]).

The relatively less restrictive representation of the fractional derivative according to parameters appears to be the one based on Pochhammer's contour integral introduced by Tremblay $[29,30]$.

Definition 1 Let $f(z)$ be analytic in a simply connected region $\mathcal{R}$ of the complex $z$-plane. Let $g(z)$ be regular and univalent on $\mathcal{R}$ and let $g^{-1}(0)$ be an interior point of $\mathcal{R}$. Then, if $\alpha$ is not a negative integer, $p$ is not an integer, and $z$ is in $\mathcal{R} \backslash\left\{g^{-1}(0)\right\}$, we define the fractional derivative of order $\alpha$ of $g(z)^{p} f(z)$ with respect to $g(z)$ by

$$
\begin{aligned}
& D_{g(z)}^{\alpha}\left\{[g(z)]^{p} f(z)\right\} \\
& \quad=\frac{\mathrm{e}^{-\mathrm{i} \pi p} \Gamma(1+\alpha)}{4 \pi \sin (\pi p)} \int_{C\left(z+g^{-1}(0)+, z-g^{-1}(0)-; F(a), F(a)\right)} \frac{f(\xi)[g(\xi)]^{p} g^{\prime}(\xi)}{[g(\xi)-g(z)]^{\alpha+1}} \mathrm{~d} \xi .
\end{aligned}
$$

For non-integers $\alpha$ and $p$, the functions $g(\xi)^{p}$ and $[g(\xi)-g(z)]^{-\alpha-1}$ in the integrand have two branch lines which begin, respectively, at $\xi=z$ and $\xi=g^{-1}(0)$, and both branches pass through the point $\xi=a$ without crossing the Pochhammer contour $P(a)=\left\{C_{1} \cup C_{2} \cup C_{3} \cup\right.$ $\left.C_{4}\right\}$ at any other point as shown in Figure 1. Here $F(a)$ denotes the principal value of the integrand in (2.3) at the beginning and the ending point of the Pochhammer contour $P(a)$ which is closed on the Riemann surface of the multiple-valued function $F(\xi)$.

Remark 1 In Definition 1, the function $f(z)$ must be analytic at $\xi=g^{-1}(0)$. However, it is interesting to note here that, if we could also allow $f(z)$ to have an essential singularity at $\xi=g^{-1}(0)$, then (2.3) would still be valid. 


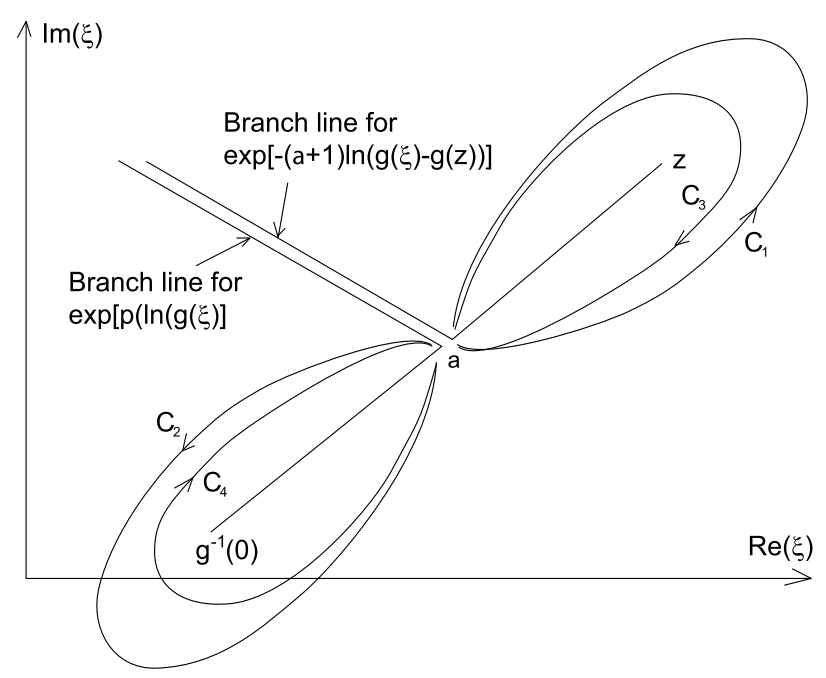

Figure 1 Pochhammer's contour.

Remark 2 In case the Pochhammer contour never crosses the singularities at $\xi=g^{-1}(0)$ and $\xi=z$ in (2.3), then we know that the integral is analytic for all $p$ and for all $\alpha$ and for $z$ in $\mathcal{R} \backslash\left\{g^{-1}(0)\right\}$. Indeed, in this case, the only possible singularities of $D_{g(z)}^{\alpha}\left\{[g(z)]^{p} f(z)\right\}$ are $\alpha=-1,-2,-3, \ldots$ and $p=0, \pm 1, \pm 2, \ldots$, which can directly be identified from the coefficient of the integral (2.3). However, by integrating by parts $N$ times the integral in (2.3) by two different ways, we can show that $\alpha=-1,-2, \ldots$ and $p=0,1,2, \ldots$ are removable singularities (see, for details, [29]).

It is well known that $[20$, p.83, Eq. (2.4)]

$$
D_{z}^{\alpha}\left\{z^{p}\right\}=\frac{\Gamma(1+p)}{\Gamma(1+p-\alpha)} z^{p-\alpha} \quad(\Re(p)>-1) .
$$

Adopting the Pochhammer-based representation for the fractional derivative modifies the restriction to the case when $p$ not a negative integer.

In their work, Srivastava et al. [10] (see also the works of Garg et al. [31] and Lin et al. [32]) gave the following fractional derivative formula for the function $\Phi_{\lambda, \mu ; \nu}^{(\rho, \sigma)}(z, s, a)$ :

$$
\begin{aligned}
& D_{z}^{\nu-\tau}\left\{z^{\nu-1} \Phi_{\lambda, \mu ; \nu}^{(\rho, \sigma, \kappa)}\left(z^{\kappa}, s, a\right)\right\}=\frac{\Gamma(\nu)}{\Gamma(\tau)} z^{\tau-1} \Phi_{\lambda, \mu ; \tau}^{(\rho, \sigma, \kappa)}\left(z^{\kappa}, s, a\right) \\
& (\Re(v)>0 ; \kappa>0) .
\end{aligned}
$$

These last restrictions become $\kappa+\nu-1$ not a negative integer and $\kappa>0$ by making use of the Pochhammer-based representation for the fractional derivative.

The fractional derivative formula (2.5) can be specialized to deduce other results. As example, upon setting $\rho=\sigma=\kappa=1$ in (2.5), we obtain

$$
D_{z}^{\nu-\tau}\left\{z^{\nu-1} \Phi_{\lambda, \mu ; \nu}(z, s, a)\right\}=\frac{\Gamma(\nu)}{\Gamma(\tau)} z^{\tau-1} \Phi_{\lambda, \mu ; \tau}(z, s, a)
$$

$(v$ not a negative integer; $\kappa>0)$. 
Another fractional derivative formula that will be very useful in the present investigation is given by the next formula:

$$
D_{z}^{\alpha}\left\{z^{\beta} \Phi_{\lambda, \mu ; \nu}^{(\rho, \sigma)}(z, s, a)\right\}=\frac{\Gamma(1+\beta)}{\Gamma(1+\beta-\alpha)} z^{\beta-\alpha} \Phi_{\lambda, \mu, 1+\beta ; \nu, 1+\beta-\alpha}^{(\rho, \sigma, 1, \kappa, 1)}(z, s, a)
$$

( $\beta$ not a negative integer),

where the Hurwitz-Lerch zeta function $\Phi_{\lambda, \mu, 1+\beta ; \nu, 1+\beta-\alpha}^{(\rho, \sigma, 1, \kappa, 1)}(z, s, a)$ occurring in (2.7) is a specialized case of the multiparameters extension of the generalized Hurwitz-Lerch zeta function defined in (1.18).

\section{Important results involving fractional calculus}

In this section, we recall five very important theorems related to fractional calculus that will play central roles in our work. Each of these theorems is the generalized Leibniz rules for fractional derivatives and the Taylor-like expansions in terms of different types of functions.

First of all, we give two generalized Leibniz rules for fractional derivatives. Theorem 1 is a slightly modified theorem obtained in 1970 by Osler [26]. Theorem 2 was given, some years ago, by Tremblay et al. [33] with the help of the properties of Pochhammer's contour representation for fractional derivatives.

Theorem 1 (i) Let $\mathcal{R}$ be a simply connected region containing the origin. (ii) Let $u(z)$ and $v(z)$ satisfy the conditions of Definition 1 for the existence of the fractional derivative. Then, for $\Re(p+q)>-1$ and $\gamma \in \mathbb{C}$, the following Leibniz rule holds true:

$$
D_{z}^{\alpha}\left\{z^{p+q} u(z) v(z)\right\}=\sum_{n=-\infty}^{\infty}\left(\begin{array}{c}
\alpha \\
\gamma+n
\end{array}\right) D_{z}^{\alpha-\gamma-n}\left\{z^{p} u(z)\right\} D_{z}^{\gamma+n}\left\{z^{q} v(z)\right\} .
$$

Theorem 2 (i) Let $\mathcal{R}$ be a simply connected region containing the origin. (ii) Let $u(z)$ and $v(z)$ satisfy the conditions of Definition 1 for the existence of the fractional derivative. (iii) Let $\mathcal{U} \subset \mathcal{R}$ be the region of analyticity of the function $u(z)$ and $\mathcal{V} \subset \mathcal{R}$ be the region of analyticity of the function $v(z)$. Then, for

$$
z \neq 0, \quad z \in \mathcal{U} \cap \mathcal{V} \text { and } \Re(1-\beta)>0,
$$

the following product rule holds true:

$$
\begin{aligned}
D_{z}^{\alpha}\left\{z^{\alpha+\beta-1} u(z) v(z)\right\}= & \frac{z \Gamma(1+\alpha) \sin (\beta \pi) \sin (\mu \pi) \sin [(\alpha+\beta-\mu) \pi]}{\sin [(\alpha+\beta) \pi] \sin [(\beta-\mu-v) \pi] \sin [(\mu+v) \pi]} \\
& \cdot \sum_{n=-\infty}^{\infty} \frac{D_{z}^{\alpha+v+1-n}\left\{z^{\alpha+\beta-\mu-1-n} u(z)\right\} D_{z}^{-1-v+n}\left\{z^{\mu-1+n} v(z)\right\}}{\Gamma(2+\alpha+v-n) \Gamma(-v+n)} .
\end{aligned}
$$

Next, in the year 1971, Osler [34] obtained the following generalized Taylor-like series expansion involving fractional derivatives.

Theorem 3 Let $f(z)$ be an analytic function in a simply connected region $\mathcal{R}$. Let $\alpha$ and $\gamma$ be arbitrary complex numbers and

$$
\theta(z)=\left(z-z_{0}\right) q(z)
$$


with $q(z)$ a regular and univalent function without any zero in $\mathcal{R}$. Let a be a positive real number and

$$
K=\{0,1, \ldots,[c]([c] \text { the largest integer not greater than } c)\} .
$$

Let $b$ and $z_{0}$ be two points in $\mathcal{R}$ such that $b \neq z_{0}$ and let

$$
\omega=\exp \left(\frac{2 \pi \mathrm{i}}{a}\right)
$$

Then the following relationship holds true:

$$
\begin{aligned}
& \sum_{k \in K} c^{-1} \omega^{-\gamma k} f\left(\theta^{-1}\left(\theta(z) \omega^{k}\right)\right) \\
& \quad=\left.\sum_{n=-\infty}^{\infty} \frac{[\theta(z)]^{c n+\gamma}}{\Gamma(c n+\gamma+1)} \cdot D_{z-b}^{c n+\gamma}\left\{f(z) \theta^{\prime}(z)\left(\frac{z-z_{0}}{\theta(z)}\right)^{c n+\gamma+1}\right\}\right|_{z=z_{0}} \quad\left(\left|z-z_{0}\right|=\left|z_{0}\right|\right)
\end{aligned}
$$

In particular, if $0<c \leqq 1$ and $\theta(z)=\left(z-z_{0}\right)$, then $k=0$ and (3.3) reduces to the following form:

$$
f(z)=\left.c \sum_{n=-\infty}^{\infty} \frac{\left(z-z_{0}\right)^{c n+\gamma}}{\Gamma(c n+\gamma+1)} D_{z-b}^{c n+\gamma}\{f(z)\}\right|_{z=z_{0}} .
$$

Equation (3.4) is usually referred to as the Taylor-Riemann formula and has been studied in several papers [27, 35-38].

We next recall that Tremblay et al. [39] discovered the power series of an analytic function $f(z)$ in terms of the rational expression $\left(\frac{z-z_{1}}{z-z_{2}}\right)$, where $z_{1}$ and $z_{2}$ are two arbitrary points inside the region $\mathcal{R}$ of analyticity of $f(z)$. In particular, they obtained the following result.

Theorem 4 (i) Let $c$ be real and positive and let

$$
\omega=\exp \left(\frac{2 \pi \mathrm{i}}{a}\right)
$$

(ii) Let $f(z)$ be analytic in the simply connected region $\mathcal{R}$ with $z_{1}$ and $z_{2}$ being interior points of $\mathcal{R}$. (iii) Let the set of curves

$$
\{C(t): C(t) \subset \mathcal{R} \text { and } 0<t \leqq r\}
$$

be defined by

$$
C(t)=C_{1}(t) \cup C_{2}(t)=\left\{z:\left|\lambda_{t}\left(z_{1}, z_{2} ; z\right)\right|=\left|\lambda_{t}\left(z_{1}, z_{2} ; \frac{z_{1}+z_{2}}{2}\right)\right|\right\},
$$

where

$$
\lambda_{t}\left(z_{1}, z_{2} ; z\right)=\left[z-\frac{z_{1}+z_{2}}{2}+t\left(\frac{z_{1}-z_{2}}{2}\right)\right] \cdot\left[z-\left(\frac{z_{1}+z_{2}}{2}\right)-t\left(\frac{z_{1}-z_{2}}{2}\right)\right],
$$




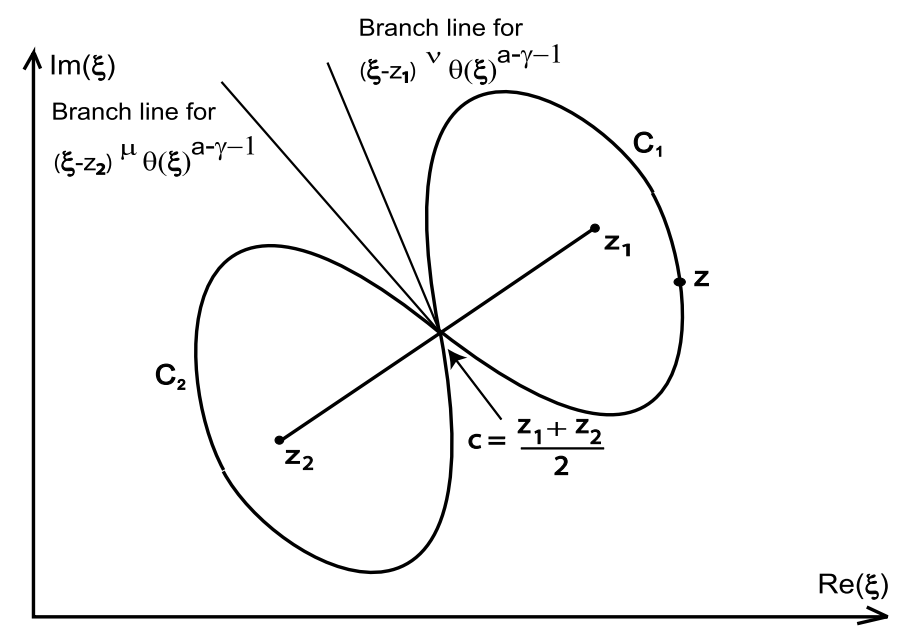

Figure 2 Multi-loops contour

which are the Bernoulli type lemniscates (see Figure 2 ) with center located at $\frac{z_{1}+z_{2}}{2}$ and with double-loops in which one loop $C_{1}(t)$ leads around the focus point

$$
\frac{z_{1}+z_{2}}{2}+\left(\frac{z_{1}-z_{2}}{2}\right) t
$$

and the other loop $C_{2}(t)$ encircles the focus point

$$
\frac{z_{1}+z_{2}}{2}-\left(\frac{z_{1}-z_{2}}{2}\right) t
$$

for each $t$ such that $0<t \leqq r$. (iv) Let

$$
\left[\left(z-z_{1}\right)\left(z-z_{2}\right)\right]^{\lambda}=\exp \left(\lambda \ln \left(\theta\left(\left(z-z_{1}\right)\left(z-z_{2}\right)\right)\right)\right)
$$

denote the principal branch of that function which is continuous and inside $C(r)$, cut by the respective two branch lines $L_{ \pm}$defined by

$$
L_{ \pm}= \begin{cases}\left\{z: z=\frac{z_{1}+z_{2}}{2} \pm t\left(\frac{z_{1}-z_{2}}{2}\right)\right\} & (0 \leqq t \leqq 1) \\ \left\{z: z=\frac{z_{1}+z_{2}}{2} \pm \mathrm{i} t\left(\frac{z_{1}-z_{2}}{2}\right)\right\} & (t<0)\end{cases}
$$

such that $\ln \left(\left(z-z_{1}\right)\left(z-z_{2}\right)\right)$ is real when $\left(z-z_{1}\right)\left(z-z_{2}\right)>0$. (v) Let $f(z)$ satisfy the conditions of Definition 1 for the existence of the fractional derivative of $\left(z-z_{2}\right)^{p} f(z)$ of order $\alpha$ for $z \in$ $\mathcal{R} \backslash\left\{L_{+} \cup L_{-}\right\}$, denoted by $D_{z-z_{2}}^{\alpha}\left\{\left(z-z_{2}\right)^{p} f(z)\right\}$, where $\alpha$ and $p$ are real or complex numbers. (vi) Let

$$
\begin{aligned}
K= & \left\{k: k \in \mathbb{N} \text { and } \arg \left(\lambda_{t}\left(z_{1}, z_{2}, \frac{z_{1}+z_{2}}{2}\right)\right)\right. \\
& <\arg \left(\lambda_{t}\left(z_{1}, z_{2}, \frac{z_{1}+z_{2}}{2}\right)\right)+\frac{2 \pi k}{a} \\
& \left.<\arg \left(\lambda_{t}\left(z_{1}, z_{2}, \frac{z_{1}+z_{2}}{2}\right)\right)+2 \pi\right\} .
\end{aligned}
$$


Then, for arbitrary complex numbers $\mu, v, \gamma$, and for $z$ on $C_{1}(1)$ defined by

$$
\begin{gathered}
\xi=\frac{z_{1}+z_{2}}{2}+\frac{z_{1}-z_{2}}{2} \sqrt{1+\mathrm{e}^{\mathrm{i} \theta}} \quad(-\pi<\theta<\pi), \\
\sum_{k \in K} \frac{c^{-1} \omega^{-\gamma k}}{z_{1}-z_{2}} f\left(\phi^{-1}\left(\omega^{k} \phi(z)\right)\right)\left[\phi^{-1}\left(\omega^{k} \phi(z)\right)-z_{1}\right]^{\nu}\left[\phi^{-1}\left(\omega^{k} \phi(z)\right)-z_{2}\right]^{\mu} \\
=\sum_{n=-\infty}^{\infty} \frac{\mathrm{e}^{\mathrm{i} \pi c(n+1)} \sin [(\mu+c n+\gamma) \pi]}{\sin [(\mu-c+\gamma) \pi] \Gamma(1-v+c n+\gamma)} \\
\left.\cdot D_{z-z_{2}}^{-v+c n+\gamma}\left\{\left(z-z_{2}\right)^{\mu+c n+\gamma-1} f(z)\right\}\right|_{z=z_{1}}[\phi(z)]^{c n+\gamma},
\end{gathered}
$$

where

$$
\phi(z)=\frac{z-z_{1}}{z-z_{2}} .
$$

The case $0<c \leqq 1$ of Theorem 4 reduces to the following form:

$$
\begin{aligned}
\frac{c^{-1} f(z)\left(z-z_{1}\right)^{\nu}\left(z-z_{2}\right)^{\mu}}{\left(z_{1}-z_{2}\right)}= & \sum_{n=-\infty}^{\infty} \frac{\mathrm{e}^{\mathrm{i} \pi c(n+1)} \sin [(\mu+c n+\gamma) \pi]}{\sin [(\mu-c+\gamma) \pi] \Gamma(1-v+c n+\gamma)} \\
& \left.\cdot D_{z-z_{2}}^{-v+c n+\gamma}\left\{\left(z-z_{2}\right)^{\mu+c n+\gamma-1} f(z)\right\}\right|_{z=z_{1}}\left(\frac{z-z_{1}}{z-z_{2}}\right)^{c n+\gamma} .
\end{aligned}
$$

Tremblay and Fugère [40] developed the power series of an analytic function $f(z)$ in terms of the function $\left(z-z_{1}\right)\left(z-z_{2}\right)$, where $z_{1}$ and $z_{2}$ are two arbitrary points inside the analyticity region $\mathcal{R}$ of $f(z)$. Explicitly, they gave the following theorem.

Theorem 5 Under the assumptions of Theorem 4, the following expansion formula holds true:

$$
\begin{aligned}
& \sum_{k \in K} c^{-1} \omega^{-\gamma k}\left[\left(\frac{z_{2}-z_{1}+\sqrt{\Delta_{k}}}{2}\right)^{\alpha}\left(\frac{z_{1}-z_{2}+\sqrt{\Delta_{k}}}{2}\right)^{\beta}\right. \cdot f\left(\frac{z_{1}+z_{2}+\sqrt{\Delta_{k}}}{2}\right)-\mathrm{e}^{\mathrm{i} \pi(\alpha-\beta)} \frac{\sin [(\alpha+c-\gamma) \pi]}{\sin [(\beta+c-\gamma) \pi]} \\
&\left.\cdot\left(\frac{z_{2}-z_{1}-\sqrt{\Delta_{k}}}{2}\right)^{\alpha}\left(\frac{z_{1}-z_{2}-\sqrt{\Delta_{k}}}{2}\right)^{\beta} f\left(\frac{z_{1}+z_{2}-\sqrt{\Delta_{k}}}{2}\right)\right] \\
&=\sum_{n=-\infty}^{\infty} \frac{\sin [(\beta-c n-\gamma) \pi]}{\sin [(\beta-c-\gamma) \pi]} \frac{\mathrm{e}^{-\mathrm{i} \pi c(n+1)}[\theta(z)]^{c n+\gamma}}{\Gamma(1-\alpha+c n+\gamma)} \\
&\left.\cdot D_{z-z_{2}}^{-\alpha+c n+\gamma}\left\{\left(z-z_{2}\right)^{\beta-c n-\gamma-1}\left(\frac{\theta(z)}{\left(z-z_{2}\right)\left(z-z_{1}\right)}\right)^{-c n-\gamma-1} \theta^{\prime}(z) f(z)\right\}\right|_{z=z_{1}},
\end{aligned}
$$

where

$$
\begin{aligned}
& \Delta_{k}=\left(z_{1}-z_{2}\right)^{2}+4 V\left(\omega^{k} \theta(z)\right), \\
& V(z)=\left.\sum_{r=1}^{\infty} D_{z}^{r-1}\left\{[q(z)]^{-r}\right\}\right|_{z=0} \frac{z^{r}}{r !}
\end{aligned}
$$


and

$$
\theta(z)=\left(z-z_{1}\right)\left(z-z_{2}\right) q\left(\left(z-z_{1}\right)\left(z-z_{2}\right)\right)
$$

As a special case, if we set $0<c \leqq 1, q(z)=1\left(\theta(z)=\left(z-z_{1}\right)\left(z-z_{2}\right)\right)$, and $z_{2}=0$ in (3.11), we obtain

$$
\begin{aligned}
f(z)= & c z^{-\beta}\left(z-z_{1}\right)^{-\alpha} \sum_{n=-\infty}^{\infty} \frac{\sin [(\beta-c n-\gamma) \pi]}{\sin [(\beta+c-\gamma) \pi]} \frac{\mathrm{e}^{\mathrm{i} \pi c(n+1)}\left[z\left(z-z_{1}\right)\right]^{c n+\gamma}}{\Gamma(1-\alpha+c n+\gamma)} \\
& \left.\cdot D_{z}^{-\alpha+c n+\gamma}\left\{z^{\beta-c n-\gamma-1}\left(z+w-z_{1}\right) f(z)\right\}\right|_{\substack{z=z_{1} \\
(w=z)}} \cdot
\end{aligned}
$$

\section{A set of main results for the generalized Hurwitz-Lerch zeta function $\Phi_{\lambda, \mu ; v}^{(\rho, \sigma, \kappa)}(z, s, a)$}

In this section, we present the new expansion formulas involving the generalized HurwitzLerch zeta functions $\Phi_{\lambda, \mu ; \nu}^{(\rho, \sigma, \kappa)}(z, s, a)$.

Theorem 6 Under the assumptions of Theorem 1, the following expansion holds true:

$$
\begin{aligned}
\Phi_{\lambda, \mu ; \tau}^{(\rho, \sigma, \kappa)}\left(z^{\kappa}, s, a\right)= & \frac{\Gamma(\tau) \Gamma(1+\nu-\tau) \sin (\gamma \pi)}{\pi} \\
& \cdot \sum_{n=-\infty}^{\infty} \frac{(-1)^{n} \Phi_{\lambda, \mu, 1 ; \nu, 1-\gamma-n}^{(\rho, \sigma, \kappa, \kappa, \kappa)}\left(z^{\kappa}, s, a\right)}{(\gamma+n) \Gamma(1+v-\tau-\gamma-n) \Gamma(\tau+\gamma+n)},
\end{aligned}
$$

provided that both members of (4.1) exist.

Proof Setting $u(z)=z^{\nu-1}$ and $v(z)=\Phi_{\lambda, \mu ; \nu}^{(\rho, \sigma, \kappa)}\left(z^{\kappa}, s, a\right)$ in Theorem 1 with $p=q=0$ and $\alpha=$ $v-\tau$, we obtain

$$
\begin{aligned}
& D_{z}^{\nu-\tau}\left\{z^{\nu-1} \Phi_{\lambda, \mu ; \nu}^{(\rho, \sigma, \kappa)}\left(z^{\kappa}, s, a\right)\right\} \\
& \quad=\sum_{n=-\infty}^{\infty}\left(\begin{array}{c}
\nu-\tau \\
\gamma+n
\end{array}\right) D_{z}^{\nu-\tau-\gamma-n}\left\{z^{\nu-1}\right\} D_{z}^{\gamma+n}\left\{\Phi_{\lambda, \mu ; \nu}^{(\rho, \sigma, \kappa)}\left(z^{\kappa}, s, a\right)\right\},
\end{aligned}
$$

which, with the help of (2.4) and (2.5), yields

$$
\begin{aligned}
& D_{z}^{\nu-\tau}\left\{z^{\nu-1} \Phi_{\lambda, \mu ; \nu}^{(\rho, \sigma, \kappa)}\left(z^{\kappa}, s, a\right)\right\}=\frac{\Gamma(\nu)}{\Gamma(\tau)} z^{\tau-1} \Phi_{\lambda, \mu ; \tau}^{(\rho, \sigma, \kappa)}\left(z^{\kappa}, s, a\right), \\
& D_{z}^{\nu-\tau-\gamma-n}\left\{z^{\nu-1}\right\}=\frac{\Gamma(\nu)}{\Gamma(\tau+\gamma+n)} z^{\tau+\gamma+n-1}
\end{aligned}
$$

and

$$
\begin{aligned}
D_{z}^{\gamma+n}\left\{\Phi_{\lambda, \mu ; \nu}^{(\rho, \sigma, \kappa)}\left(z^{\kappa}, s, a\right)\right\} & =\sum_{j=0}^{\infty} \frac{(\lambda)_{\rho j}(\mu)_{\sigma j}}{(\nu)_{\kappa j} j !} \frac{D_{z}^{\gamma+n}\left\{z^{\kappa j}\right\}}{(j+a)^{s}} \\
& =\frac{z^{-\gamma-n}}{\Gamma(1-\gamma-n)} \Phi_{\lambda, \mu, 1 ; \nu, 1-\gamma-n}^{(\rho, \sigma, \kappa, \kappa, \kappa)}\left(z^{\kappa}, s, a\right) .
\end{aligned}
$$


Combining (4.3), (4.4), (4.5) with (4.2) and making some elementary simplifications, the asserted result (4.1) follows.

Theorem 7 Under the hypotheses of Theorem 2, the following expansion formula holds true:

$$
\begin{aligned}
& \Phi_{\lambda, \mu ; \tau}^{(\rho, \sigma, \kappa)}\left(z^{\kappa}, s, a\right) \\
&=\frac{\Gamma(\tau) \Gamma(1+v-\tau) \sin (\beta \pi) \sin [(v-\tau+\beta-\theta) \pi]}{\Gamma(v) \Gamma(\tau-\gamma-\theta-1) \Gamma(1+\gamma+\theta) \sin [(\nu-\tau+\beta) \pi] \sin [(\beta-\theta-\gamma) \pi]} \\
& \cdot \frac{\sin (\theta \pi)}{\sin [(\theta+\gamma) \pi]} \sum_{n=-\infty}^{\infty} \frac{\Gamma(\nu-\theta-n) \Gamma(\theta+n)}{\Gamma(2+v-\tau+\gamma-n) \Gamma(-\gamma+n)} \\
& \cdot \Phi_{\lambda, \mu, \theta+n ; \nu, 1+\theta+\gamma}^{(\rho, \sigma, \kappa)}\left(z^{\kappa}, s, a\right),
\end{aligned}
$$

provided that both members of (4.6) exist.

Proof Upon first substituting $\mu \mapsto \theta$ and $\nu \mapsto \gamma$ in Theorem 2 and then setting

$$
\alpha=v-\tau, \quad u(z)=z^{\tau-\beta} \quad \text { and } \quad v(z)=\Phi_{\lambda, \mu ; \nu}^{(\rho, \sigma, \kappa)}\left(z^{\kappa}, s, a\right),
$$

in which both $u(z)$ and $v(z)$ satisfy the conditions of Theorem 2, we have

$$
\begin{aligned}
D_{z}^{\nu-\tau} & \left\{z^{\nu-1} \Phi_{\lambda, \mu ; \nu}^{(\rho, \sigma, \kappa)}\left(z^{\kappa}, s, a\right)\right\} \\
= & \frac{z \Gamma(1+\nu-\tau) \sin (\beta \pi) \sin (\theta \pi) \sin [(\nu-\tau+\beta-\theta) \pi]}{\sin [(\nu-\tau+\beta) \pi] \sin [(\beta-\theta-\gamma) \pi] \sin [(\theta+\gamma) \pi]} \\
& \quad \sum_{n=-\infty}^{\infty} \frac{D_{z}^{\nu-\tau+\gamma+1-n}\left\{z^{\nu-\theta-1-n}\right\} D_{z}^{-1-\gamma+n}\left\{z^{\theta-1+n} \Phi_{\lambda, \mu ; \nu}^{(\rho, \sigma, \kappa)}\left(z^{\kappa}, s, a\right)\right\}}{\Gamma(2+\nu-\tau+\gamma-n) \Gamma(-\gamma+n)} .
\end{aligned}
$$

Now, by using (2.4) and (2.5), we find that

$$
\begin{aligned}
& D_{z}^{\nu-\tau}\left\{z^{\nu-1} \Phi_{\lambda, \mu ; \nu}^{(\rho, \sigma, \kappa)}\left(z^{\kappa}, s, a\right)\right\}=\frac{\Gamma(\nu)}{\Gamma(\tau)} z^{\tau-1} \Phi_{\lambda, \mu ; \tau}^{(\rho, \sigma, \kappa)}\left(z^{\kappa}, s, a\right), \\
& D_{z}^{\nu-\tau+\gamma+1-n}\left\{z^{\nu-\theta-1-n}\right\}=\frac{\Gamma(\nu-\theta-n)}{\Gamma(\tau-\gamma-\theta-1)} z^{\tau-\gamma-\theta-2}
\end{aligned}
$$

and

$$
\begin{aligned}
& D_{z}^{-1-\gamma+n}\left\{z^{\theta-1+n} \Phi_{\lambda, \mu ; \nu}^{(\rho, \sigma, \kappa)}\left(z^{\kappa}, s, a\right)\right\} \\
& \quad=\frac{\Gamma(\theta+n)}{\Gamma(1+\theta+\gamma)} z^{\theta+\gamma} \Phi_{\lambda, \mu, \theta+n ; \nu, 1+\theta+\gamma}^{(\rho, \sigma, \kappa, \kappa, \kappa)}\left(z^{\kappa}, s, a\right) .
\end{aligned}
$$

Thus, finally, the result (4.6) follows by combining (4.8), (4.9), (4.10), and (4.7).

We now shift our focus on the different Taylor-like expansions in terms of different types of functions involving the generalized Hurwitz-Lerch zeta functions $\Phi_{\lambda, \mu ; \nu}^{(\rho, \sigma, \kappa)}(z, s, a)$. 
Theorem 8 Under the assumptions of Theorem 3, the following expansion formula holds true:

$$
\begin{gathered}
\Phi_{\lambda, \mu ; \nu}^{(\rho, \sigma, \kappa)}(z, s, a)=c \sum_{n=-\infty}^{\infty} \frac{z_{0}^{-c n}\left(z-z_{0}\right)^{c n}}{\Gamma(c n+1) \Gamma(1-c n)} \Phi_{\lambda, \mu, 1 ; v, 1-c n}^{(\rho, \sigma, 1, \kappa, 1)}\left(z_{0}, s, a\right) \\
\left(\left|z-z_{0}\right|=\left|z_{0}\right| ; \lambda>0\right),
\end{gathered}
$$

provided that both members of (4.11) exist.

Proof Setting $f(z)=\Phi_{\lambda, \mu ; \nu}^{(\rho, \sigma, \kappa)}(z, s, a)$ in Theorem 3 with $b=\gamma=0,0<c \leqq 1$, and $\theta(z)=z-z_{0}$, we have

$$
\Phi_{\lambda, \mu ; \nu}^{(\rho, \sigma, \kappa)}(z, s, a)=\left.c \sum_{n=-\infty}^{\infty} \frac{\left(z-z_{0}\right)^{c n}}{\Gamma(1+c n)} D_{z}^{c n}\left\{\Phi_{\lambda, \mu ; \nu}^{(\rho, \sigma, \kappa)}(z, s, a)\right\}\right|_{z=z_{0}}
$$

for $z_{0} \neq 0$ and for $z$ such that $\left|z-z_{0}\right|=\left|z_{0}\right|$.

Now, by making use of (2.7) with $\beta=0$ and $\alpha=c n$, we find that

$$
\left.D_{z}^{c n}\left\{\Phi_{\lambda, \mu ; \nu}^{(\rho, \sigma, \kappa)}(z, s, a)\right\}\right|_{z=z_{0}}=\frac{z_{0}^{-c n}}{\Gamma(1-c n)} \Phi_{\lambda, \mu, 1 ; \nu, 1-c n}^{(\rho, \sigma, 1, \mu, 1)}\left(z_{0}, s, a\right) .
$$

By combining (4.12) and (4.13), we get the result (4.11) asserted by Theorem 8 .

Theorem 9 Under the hypotheses of Theorem 4, the following expansion formula holds true:

$$
\begin{aligned}
\Phi_{\lambda, \mu ; \nu}^{(\rho, \sigma, \kappa)}(z, s, a)= & c z^{-\alpha}\left(z-z_{1}\right)^{-\beta} z_{1}^{\alpha+\beta} \\
& \cdot \sum_{n=-\infty}^{\infty} \frac{\mathrm{e}^{\mathrm{i} \pi c(n+1)} \sin [(\alpha+c n+\gamma) \pi] \Gamma(\alpha+c n+\gamma)}{\sin [(\alpha-c+\gamma) \pi] \Gamma(1-\beta+c n+\gamma) \Gamma(\alpha+\beta)} \\
& \cdot \Phi_{\lambda, \mu, \alpha+c n+\gamma ; \nu, \alpha+\beta}^{(\rho, \sigma, 1, \alpha, 1)}\left(z_{1}, s, a\right)\left(\frac{z-z_{1}}{z}\right)^{c n+\gamma}
\end{aligned}
$$

for $\lambda>0$ and for $z$ on $C_{1}(1)$ defined by

$$
z=\frac{z_{1}}{2}+\frac{z_{1}}{2} \sqrt{1+\mathrm{e}^{\mathrm{i} \theta}} \quad(-\pi<\theta<\pi)
$$

provided that both sides of (4.14) exist.

Proof By taking $f(z)=\Phi_{\lambda, \mu ; \nu}^{(\rho, \sigma, \kappa)}(z, s, a)$ in Theorem 4 with $z_{2}=0, \mu=\alpha, \nu=\beta$, and $0<c \leqq 1$, we find that

$$
\begin{aligned}
\Phi_{\lambda, \mu ; \nu}^{(\rho, \sigma, \kappa)}(z, s, a)= & c\left(z-z_{1}\right)^{-\beta} z^{-\alpha} z_{1} \\
& \cdot \sum_{n=-\infty}^{\infty} \frac{\mathrm{e}^{\mathrm{i} \pi c(n+1)} \sin [(\alpha+c n+\gamma) \pi]}{\sin [(\alpha-c+\gamma) \pi] \Gamma(1-\beta+c n+\gamma)} \\
& \left.\cdot D_{z}^{-\beta+c n+\gamma}\left\{z^{\alpha+c n+\gamma-1} \Phi_{\lambda, \mu ; \nu}^{(\rho, \sigma, \kappa)}(z, s, a)\right\}\right|_{z=z_{1}}\left(\frac{z-z_{1}}{z}\right)^{c n+\gamma} .
\end{aligned}
$$


Now, with the help of the relation (2.7) with $\alpha \mapsto-\beta+c n+\gamma$ and $\beta \mapsto \alpha+c n+\gamma-1$, we have

$$
\begin{aligned}
& \left.D_{z}^{-\beta+c n+\gamma}\left\{z^{\alpha+c n+\gamma-1} \Phi_{\lambda, \mu ; \nu}^{(\rho, \sigma, \kappa)}(z, s, a)\right\}\right|_{z=z_{1}} \\
& =z_{1}^{\alpha+\beta-1} \frac{\Gamma(\alpha+c n+\gamma)}{\Gamma(\alpha+\beta)} \Phi_{\lambda, \mu, \alpha+c n+\gamma ; \nu, \alpha+\beta}^{(\rho, \sigma, 1, \kappa, 1)}\left(z_{1}, s, a\right) .
\end{aligned}
$$

Thus, by combining (4.15) and (4.16), we are led to the assertion (4.14) of Theorem 9.

Theorem 10 Under the hypotheses of Theorem 5, the following expansion formula holds true:

$$
\begin{aligned}
\Phi_{\lambda, \mu ; \nu}^{(\rho, \sigma, \kappa)}(z, s, a)= & c z^{-\beta+\gamma}\left(z-z_{1}\right)^{-\alpha+\gamma} z_{1}^{\beta+\alpha-2 \gamma-1} \\
& \cdot \sum_{n=-\infty}^{\infty} \frac{\sin [(\beta-c n-\gamma) \pi] \mathrm{e}^{\mathrm{i} \pi c(n+1)}}{\sin [(\beta+c-\gamma) \pi] \Gamma(1-\alpha+c n+\gamma)}\left(\frac{z\left(z-z_{1}\right)}{z_{1}^{2}}\right)^{c n} \\
& \cdot \frac{\Gamma(\beta-c n-\gamma)}{\Gamma(\beta+\alpha-2 c n-2 \gamma)}\left[\left(z-z_{1}\right) \Phi_{\lambda, \mu, \beta-c n-\gamma ; \nu, \beta+\alpha-2 c n-2 \gamma}^{(\rho, \sigma, 1, \kappa, 1)}\left(z_{1}, s, a\right)\right. \\
& \left.+\left(\frac{\beta-c n-\gamma}{\alpha+\beta-2 c n-2 \gamma}\right) z_{1} \Phi_{\lambda, \mu, 1+\beta-c n-\gamma ; \nu, 1+\beta+\alpha-2 c n-2 \gamma}^{(\rho, \sigma, 1, \kappa, 1)}\left(z_{1}, s, a\right)\right]
\end{aligned}
$$

for $\lambda>0$ and for $z$ on $C_{1}(1)$ defined by

$$
z=\frac{z_{1}}{2}+\frac{z_{1}}{2} \sqrt{1+\mathrm{e}^{\mathrm{i} \theta}} \quad(-\pi<\theta<\pi)
$$

provided that both sides of (4.17) exist.

Proof Putting $f(z)=\Phi_{\lambda, \mu ; \nu}^{(\rho, \sigma)}(z, s, a)$ in Theorem 5 with $z_{2}=0,0<c \leqq 1, q(z)=1$, and $\theta(z)=$ $\left(z-z_{1}\right)\left(z-z_{2}\right)$, we find that

$$
\begin{aligned}
\Phi_{\lambda, \mu ; \nu}^{(\rho, \sigma, \kappa)}(z, s, a)= & c z^{-\beta}\left(z-z_{1}\right)^{-\alpha} \sum_{n=-\infty}^{\infty} \frac{\sin [(\beta-c n-\gamma) \pi]}{\sin [(\beta+c-\gamma) \pi]} \frac{\mathrm{e}^{\mathrm{i} \pi c(n+1)}\left[z\left(z-z_{1}\right)\right]^{c n+\gamma}}{\Gamma(1-\alpha+c n+\gamma)} \\
& \left.\cdot D_{z}^{-\alpha+c n+\gamma}\left\{z^{\beta-c n-\gamma-1}\left(z+w-z_{1}\right) \Phi_{\lambda, \mu ; \nu}^{(\rho, \sigma)}(z, s, a)\right\}\right|_{\substack{z=z_{1} \\
(w=z)}} \cdot
\end{aligned}
$$

With the help of relation (2.7), we have

$$
\begin{aligned}
\left.D_{z}^{-\alpha+c n+\gamma}\left\{z^{\beta-c n-\gamma-1}\left(z+w-z_{1}\right) \Phi_{\lambda, \mu ; \nu}^{(\rho, \sigma, \kappa)}(z, s, a)\right\}\right|_{\substack{z=z_{1} \\
(w=z)}} & \left.D_{z}^{-\alpha+c n+\gamma}\left\{z^{\beta-c n-\gamma} \Phi_{\lambda, \mu ; \nu}^{(\rho, \sigma)}(z, s, a)\right\}\right|_{z=z_{1}} \\
& \quad+\left.\left(z-z_{1}\right) D_{z}^{-\alpha+c n+\gamma}\left\{z^{\beta-c n-\gamma-1} \Phi_{\lambda, \mu ; \nu}^{(\rho, \sigma, \kappa)}(z, s, a)\right\}\right|_{z=z_{1}} \\
= & z_{1}^{\beta+\alpha-2 c n-2 \gamma}\left(\frac{\Gamma(1+\beta-c n-\gamma)}{\Gamma(1+\beta+\alpha-2 c n-2 \gamma)} \Phi_{\lambda, \mu, 1+\beta-c n-\gamma ; \nu, 1+\beta+\alpha-2 c n-2 \gamma}^{(\rho, \sigma, 1, \kappa, 1)}\left(z_{1}, s, a\right)\right. \\
& \left.\quad+\left(\frac{z-z_{1}}{z_{1}}\right) \frac{\Gamma(\beta-c n-\gamma)}{\Gamma(\beta+\alpha-2 c n-2 \gamma)} \Phi_{\lambda, \mu, \beta-c n-\gamma ; \nu, \beta+\alpha-2 c n-2 \gamma}^{(\rho, \sigma, 1, \kappa, 1)}\left(z_{1}, s, a\right)\right) .
\end{aligned}
$$

Thus, by combining (4.18) and (4.19), we obtain the desired result (4.17). 


\section{Corollaries and consequences}

This section is devoted to the presentation of some special cases of the main results. These special cases and consequences are given in the form of the following corollaries.

Setting $\mu=\rho=\sigma=1$ in Theorem 6 with $z \mapsto\left(\frac{z}{\lambda}\right)^{1 / \kappa}$, dividing by $\Gamma(v)$ and taking the limit when $\lambda \rightarrow \infty$, we deduce the following expansion formula.

Corollary 1 Under the hypotheses of Theorem 6, the following expansion holds true:

$$
\begin{aligned}
E_{\kappa, \tau}^{(a)}(s ; z)= & \frac{\Gamma(\tau) \Gamma(1+v-\tau) \sin (\gamma \pi)}{\pi \Gamma(v)} \\
& \cdot \sum_{n=-\infty}^{\infty} \frac{(-1)^{n} \Phi_{1,1 ; i, 1,-\gamma-n}^{(1, \kappa, \kappa, \kappa)}(z, s, a)}{(\gamma+n) \Gamma(1+v-\tau-\gamma-n) \Gamma(\tau+\gamma+n)}
\end{aligned}
$$

provided that both members of (5.1) exist.

Letting $\rho=\sigma=\kappa=1$ in Theorem 7 leads to the following expansion formula.

Corollary 2 Under the assumptions of Theorem 7, the following expansion formula holds true:

$$
\begin{aligned}
\Phi_{\lambda, \mu ; \tau}(z, s, a) & \frac{\Gamma(\tau) \Gamma(1+v-\tau) \sin \beta \pi \sin (v-\tau+\beta-\theta) \pi}{\Gamma(v) \Gamma(\tau-\gamma-\theta-1) \Gamma(1+\gamma+\theta) \sin (v-\tau+\beta) \pi \sin (\beta-\theta-\gamma) \pi} \\
& \cdot \frac{\sin \theta \pi}{\sin (\theta+\gamma) \pi} \sum_{n=-\infty}^{\infty} \frac{\Gamma(v-\theta-n) \Gamma(\theta+n)}{\Gamma(2+v-\tau+\gamma-n) \Gamma(-\gamma+n)} \\
& \cdot \Phi_{\lambda, \mu, \theta+n ; \nu, 1+\theta+\gamma}^{(1,1,1,1)}(z, s, a)
\end{aligned}
$$

provided that both members of (5.2) exist.

Putting $\rho=\sigma=\kappa=1$ and replacing $\lambda$ by $\nu$ in Theorem 9, we deduce the following expansion formula given recently by Gaboury [17, Eq. (4.4)].

Corollary 3 Under the hypotheses of Theorem 9, the following expansion formula holds true:

$$
\begin{aligned}
\Phi_{\mu}^{*}(z, s, a)= & c z^{-\alpha}\left(z-z_{1}\right)^{-\beta} z_{1}^{\alpha+\beta} \sum_{n=-\infty}^{\infty} \frac{\mathrm{e}^{\mathrm{i} \pi c(n+1)} \sin [(\alpha+c n+\gamma) \pi] \Gamma(\alpha+c n+\gamma)}{\sin [(\alpha-c+\gamma) \pi] \Gamma(1-\beta+c n+\gamma) \Gamma(\alpha+\beta)} \\
& \cdot \Phi_{\mu, \alpha+c n+\gamma ; \alpha+\beta}^{(1,1,1)}\left(z_{1}, s, a\right)\left(\frac{z-z_{1}}{z}\right)^{c n+\gamma}
\end{aligned}
$$

for $z$ on $C_{1}(1)$ defined by

$$
z=\frac{z_{1}}{2}+\frac{z_{1}}{2} \sqrt{1+\mathrm{e}^{\mathrm{i} \theta}} \quad(-\pi<\theta<\pi)
$$

provided that both sides of (5.3) exist. 
Setting $\lambda=\rho=1$ in Theorem 10, we obtain the following corollary.

Corollary 4 Under the hypotheses of Theorem 10, the following expansion holds true:

$$
\begin{aligned}
\Phi_{\mu, \nu}^{(\sigma, \kappa)}(z, s, a)= & c z^{-\beta+\gamma}\left(z-z_{1}\right)^{-\alpha+\gamma} z_{1}^{\beta+\alpha-2 \gamma-1} \\
& \cdot \sum_{n=-\infty}^{\infty} \frac{\sin [(\beta-c n-\gamma) \pi] \mathrm{e}^{\mathrm{i} \pi c(n+1)}}{\sin [(\beta+c-\gamma) \pi] \Gamma(1-\alpha+c n+\gamma)}\left(\frac{z\left(z-z_{1}\right)}{z_{1}^{2}}\right)^{c n} \\
& \cdot \frac{\Gamma(\beta-c n-\gamma)}{\Gamma(\beta+\alpha-2 c n-2 \gamma)}\left[\left(z-z_{1}\right) \Phi_{1, \mu, \beta-c n-\gamma ; \nu, \beta+\alpha-2 c n-2 \gamma}^{(1, \sigma, 1, \kappa, 1)}\left(z_{1}, s, a\right)\right. \\
& \left.+\left(\frac{\beta-c n-\gamma}{\alpha+\beta-2 c n-2 \gamma}\right) z_{1} \Phi_{1, \mu, 1+\beta-c n-\gamma ; \nu, 1+\beta+\alpha-2 c n-2 \gamma}^{(1, \sigma, 1, \kappa, 1)}\left(z_{1}, s, a\right)\right]
\end{aligned}
$$

for $\lambda>0$ and for $z$ on $C_{1}(1)$ defined by

$$
z=\frac{z_{1}}{2}+\frac{z_{1}}{2} \sqrt{1+\mathrm{e}^{\mathrm{i} \theta}} \quad(-\pi<\theta<\pi)
$$

provided that both sides of (5.5) exist.

In our series of forthcoming papers, we propose to consider and investigate analogous expansion formulas and other results involving the more general multi-parameter family of the Hurwitz-Lerch zeta function (1.18) and also their $\lambda$-extensions considered recently by Srivastava et al. [41] and Srivastava [42].

\section{Competing interests}

The authors declare that they have no competing interests.

Authors' contributions

All authors completed the paper together. All authors read and approved the final manuscript.

\section{Author details}

'Department of Mathematics and Statistics, University of Victoria, Victoria, British Columbia V8W 3R4, Canada. 2Department of Mathematics and Computer Science, University of Québec at Chicoutimi, Chicoutimi, Québec G7H 2B1, Canada. ${ }^{3}$ Département de Mathématiques, Université d'Evry Val D’Essonne, 23 BD de France, Evry Cedex, 91037, France.

\section{Acknowledgements}

The authors wish to thank referees for valuable suggestions and comments.

Received: 27 March 2014 Accepted: 4 June 2014 Published: 23 June 2014

\section{References}

1. Srivastava, HM, Choi, J: Series Associated with Zeta and Related Functions. Kluwer Academic, Dordrecht (2001)

2. Srivastava, HM: Some formulas for the Bernoulli and Euler polynomials at rational arguments. Math. Proc. Camb. Philos. Soc. 129, 77-84 (2000)

3. Srivastava, HM, Choi, J: Zeta and $q$-Zeta Functions and Associated Series and Integrals. Elsevier, Amsterdam (2012)

4. Ozden, H, Cangul, IN, Simsek, Y: Hurwitz type multiple Genocchi zeta function. AIP Conf. Proc. 1148, 781-784 (2009)

5. Srivastava, HM, Kurt, B, Simsek, Y: Some families of Genocchi type polynomials and their interpolation functions. Integral Transforms Spec. Funct. 23, 919-938 (2012)

6. Lin, S-D, Srivastava, HM: Some families of the Hurwitz-Lerch zeta functions and associated fractional derivative and other integral representations. Appl. Math. Comput. 154, 725-733 (2004)

7. Srivastava, HM, Manocha, HL: A Treatise on Generating Functions. Halsted, New York (1984)

8. Goyal, SP, Laddha, RK: On the generalized Riemann zeta function and the generalized Lambert transform. Ganita Sandesh 11, 99-108 (1997)

9. Garg, M, Jain, K, Kalla, SL: A further study of general Hurwitz-Lerch zeta function. Algebras Groups Geom. 25, 311-319 (2008)

10. Srivastava, HM, Saxena, RK, Pogány, TK, Saxena, R: Integral and computational representations of the extended Hurwitz-Lerch zeta function. Integral Transforms Spec. Funct. 22, 487-506 (2011)

11. Srivastava, HM: Some generalizations and basic (or q-) extensions of the Bernoulli, Euler and Genocchi polynomials. Appl. Math. Inf. Sci. 5, 390-444 (2011) 
12. Srivastava, HM: Generating relations and other results associated with some families of the extended Hurwitz-Lerch zeta functions. SpringerPlus 2, Article ID 67 (2013)

13. Srivastava, HM, Jankov, D, Pogány, TK, Saxena, RK: Two-sided inequalities for the extended Hurwitz-Lerch zeta function. Comput. Math. Appl. 62, 516-522 (2011)

14. Jankov, D, Pogány, TK, Saxena, RK: An extended general Hurwitz-Lerch zeta function as a Mathieu $(a, \lambda)$-series. Appl. Math. Lett. 24, 1473-1476 (2011)

15. Barnes, EW: The asymptotic expansion of integral functions defined by Taylor series. Philos. Trans. R. Soc. Lond. Ser. A 206, 249-297 (1906)

16. Gaboury, S, Bayad, A: Further expansion formulas for a class of generalized Hurwitz-Lerch zeta functions obtained by means of a new Leibniz rule for fractional derivatives. Preprint (2013)

17. Gaboury, S: Some relations involving generalized Hurwitz-Lerch zeta function obtained by means of fractional derivatives with applications to Apostol-type polynomials. Adv. Differ. Equ. 2013, Article ID 361 (2013)

18. Baleanu, D, Diethelm, K, Scalas, E, Trujillo, JJ: Fractional Calculus Models and Numerical Methods. Series on Complexity, Nonlinearity and Chaos. World Scientific, Boston (2012)

19. Kilbas, AA, Srivastava, HM, Trujillo, JJ: Theory and Applications of Fractional Differential Equations. North-Holland Mathematical Studies, vol. 204. Elsevier, Amsterdam (2006)

20. Miller, KS, Ross, B: An Introduction of the Fractional Calculus and Fractional Differential Equations. Wiley, New York (1993)

21. Samko, SG, Kilbas, AA, Marichev, Ol: Fractional Integrals and Derivatives: Theory and Applications. Gordon \& Breach, New York (1993)

22. Erdélyi, A: An integral equation involving Legendre polynomials. SIAM J. Appl. Math. 12, 15-30 (1964)

23. Liouville, J: Mémoire sur le calcul des différentielles à indices quelconques. J. Éc. Polytech. 13, 71-162 (1832)

24. Riesz, M: L'intégrale de Riemann-Liouville et le problème de Cauchy. Acta Math. 81, 1-222 (1949)

25. Osler, TJ: Fractional derivatives of a composite function. SIAM J. Math. Anal. 1, 288-293 (1970)

26. Osler, TJ: Leibniz rule for the fractional derivatives and an application to infinite series. SIAM J. Appl. Math. 18,658-674 (1970)

27. Osler, TJ: Leibniz Rule, the Chain Rule and Taylor's Theorem for Fractional Derivatives. PhD thesis, New York University (1970)

28. Osler, TJ: Fractional derivatives and Leibniz rule. Am. Math. Mon. 78, 645-649 (1971)

29. Lavoie, J-L, Osler, TJ, Tremblay, R: Fundamental properties of fractional derivatives via Pochhammer integrals. In: Ross, B (ed.) Fractional Calculus and Its Applications. Proceedings of the International Conference (University of New Haven; June 15-16, 1974). Lecture Notes in Mathematics, vol. 457, pp. 323-356. Springer, Berlin (1975)

30. Tremblay, R: Une Contribution à la Théorie de la Dérivée Fractionnaire. PhD thesis, Laval University, Québec (1974)

31. Garg, M, Jain, K, Srivastava, HM: Some relationships between the generalized Apostol-Bernoulli polynomials and Hurwitz-Lerch zeta functions. Integral Transforms Spec. Funct. 17, 803-815 (2006)

32. Lin, S-D, Srivastava, HM, Wang, P-Y: Some expansion formulas for a class of generalized Hurwitz-Lerch zeta functions. Integral Transforms Spec. Funct. 17, 817-827 (2006)

33. Tremblay, R, Gaboury, S, Fugère, B-J: A new Leibniz rule and its integral analogue for fractional derivatives. Integral Transforms Spec. Funct. 24, 111-128 (2013)

34. Osler, TJ: Taylor's series generalized for fractional derivatives and applications. SIAM J. Math. Anal. 2, 37-48 (1971)

35. Hardy, GH: Riemann's forms of Taylor's series. J. Lond. Math. Soc. 20, $48-57$ (1945)

36. Heaviside, O: Electromagnetic Theory, vol. 2. Dover, New York (1950)

37. Riemann, B: Versuch einer allgemeinen Auffasung der Integration und Differentiation. The Collected Works of Bernhard Riemann. Dover, New York (1953)

38. Watanabe, Y: Zum Riemanschen binomischen Lehrsatz. Proc. Phys. Math. Soc. Jpn. 14, $22-35$ (1932)

39. Tremblay, R, Gaboury, S, Fugère, B-J: Taylor-like expansion in terms of a rational function obtained by means of fractional derivatives. Integral Transforms Spec. Funct. 24, 50-64 (2013)

40. Tremblay, R, Fugère, B-J: The use of fractional derivatives to expand analytical functions in terms of quadratic functions with applications to special functions. Appl. Math. Comput. 187, 507-529 (2007)

41. Srivastava, HM, Luo, M-J, Raina, RK: New results involving a class of generalized Hurwitz-Lerch zeta functions and their applications. Turk. J. Anal. Number Theory 1, 26-35 (2013)

42. Srivastava, HM: A new family of the $\lambda$-generalized Hurwitz-Lerch zeta functions with applications. Appl. Math. Inf. Sci. $8,1485-1500(2014)$

doi:10.1186/1687-1847-2014-169

Cite this article as: Srivastava et al.: Expansion formulas for an extended Hurwitz-Lerch zeta function obtained via fractional calculus. Advances in Difference Equations 2014 2014:169. 